\title{
Uso de los datos de plumbemia para evaluar y prevenir el envenenamiento infantil por plomo en Latinoamérica
}

Isabelle Romieu, MD, MPH, ScD. (1)

\begin{abstract}
Romieu I.
Uso de los datos de plumbemia para evaluar y prevenir el envenenamiento infantil por plomo en Latinoamérica.

Salud Publica Mex 2003;45 supl 2:S244-S251

El texto completo en inglés de este artículo está disponible en: http://www.insp.mx/salud/index.html
\end{abstract}

\section{Resumen}

La exposición al plomo es una amenaza seria para la salud de los niños, que se encuentra ampliamente distribuida en la Región deAmérica Latina. Los funcionarios de salud pública necesitan identificar fuentes de exposición al plomo, así como sus efectos en la salud, para poder diseñar, implantar y evaluar las actividades preventivas y de control. Para evaluar la magnitud del problema del plomo es necesario definir tres elementos clave: a) las fuentes potenciales de exposición, b) los indicado res que se utilizarán para evaluar los efectos en la salud y la exposición en el medio ambiente, y c) las estrategias de muestreo de la población en riesgo. Se pueden utilizar varias estrategias con el fin de seleccionar la población blanco dependiendo de los objetivos del estudio, el tiempo límite y los recursos disponibles. Si el objetivo es evaluar la magnitud y las fuentes del problema pueden emplearse los siguientes métodos de muestreo:a) el muestreo al azar basado en la población; b) el muestreo al azar basado en las facilidades dentro de los hospitales, las guarderías - las escuelas; c) el muestreo objetivo de los grupos de alto riesgo;d) el muestreo de conveniencia (de voluntarios), y e) el reporte de los casos (éste puede conducir a la identificación de las poblaciones en riesgo, además de las fuentes de exposición). Para todos los diseños de muestreo, la información debe incluir tanto el uso de un cuestionario para el informe general de los participantes y de las fuentes potenciales locales de exposición, como la recolección de las muestras biológicas. En la interpretación de los datos se

\author{
Romieu I. \\ Use of blood lead data in epidemiological studies \\ to assess and prevent childhood lead poisoning \\ in Latin America. \\ Salud Publica Mex 2003;45 suppl 2:S244-S251. \\ The English version of this paper \\ is available at: http://www.insp.mx/salud/index.html
}

(1) Instituto Nacional de Salud Pública, Cuernavaca, Morelos, México.

\begin{abstract}
A bstract
Exposure to lead is a widespread and serious threat to the health of children in Latin America. Health officials should monitor sources of exposure and health outcomes to design, implement, and evaluate prevention and control activities. To evaluate the magnitude of lead as a public health problem, three key elements must be defined: 1) the potential sources of exposure, 2) the indicators to evaluate health effects and environmental exposure, and 3) the sampling methods for the population at risk. Several strategies can be used to select the study population depending on the study objectives, the time limitations, and the available resources. If the objective is to evaluate the magnitude and sources of the problem, the following sampling methods can be used: 1) population-based random sampling; 2) facility-based random sampling within hospitals, daycare centers, or schools; 3 ) target sampling of high risk groups; 4) convenience sampling of volunteers; and 5) case reporting (which can lead to the identification of populations at risk and sources of exposures). For all sampling methods, information gathering should include the use of a questionnaire to collect general information on the participants and on potential local sources of exposure, as well as the collection of biological samples. In interpreting data, one should consider the type of sampling used and the non-response rates, as well as factors that might influence blood lead measurements, such as age and seasonal variability. Blood lead measurements should be integrated in an overall strat-
\end{abstract}

Fecha de recibido: 6 de diciembre de 2002 - Fecha de aprobado: 29 de mayo de 2003 Solicitud de sobretiros: D ra. Isabelle Romieu. Instituto N acional de Salud Pública,Avenida Universidad 655, colonia Santa María A huacatitlán, 62508 Cuernavaca, Morelos, México. Correo electrónico: iromieu@ correo.insp.mx 
debe considerar el tipo de muestreo utilizado, el porcentaje de falta de respuesta y los factores que pueden influir en las mediciones del plomo en sangre, como la edad y la variación de la temporada. Las mediciones del plomo en la sangre se deben integrar dentro de una estrategia general para la prevención de toxicidad. El texto completo en inglés de este artículo está disponible en: http://www.insp.mx/salud/index.html

Palabras clave: envenenamiento por plomo/epidemiología; vigilancia epidemioló gica; niño egy to prevent lead toxicity in children. The English version of this paper is available at: http://www.insp.mx/salud/ index.html

Key words: lead poisoning/epidemiology; epidemiologic surveillance, child a exposición al plomo es una amenaza seria para la salud de los niños, ampliamente distribuida en la Región de América Latina. Los funcionarios de salud pública necesitan identificar las fuentes de exposición al plomo, así como sus consecuencias en la salud para poder diseñar, implantar y evaluar las actividades preventivas y de control. En los Estados Unidos de América (EUA) los estudios de salud basados en la población, como el Estudio Nacional sobre la Salud y la Nutrición (National Health and Nutrition Examination Survey -NHANES, por sus siglas en inglés-) proporcionan información amplia sobre la prevalencia de las condiciones crónicas y las características específicas del estado nutricional de la población, y sobre los resultados de las pruebas hechas para la detección de marcadores biológicos como los niveles de plomo en la sangre (NPS). ${ }^{1}$ Sin embargo, en la mayoría de los países de América Latina no existe un programa de investigación para la detección del envenenamiento por plomo, ni tampoco un reporte obligatorio del envenenamiento infantil. Además, los planteamientos tradicionales de vigilancia, utilizados en la práctica de la salud pública, son difíciles de aplicar para el envenenamiento por plomo, debido a que los efectos adversos relacionados con la salud no se pueden diagnosticar clínicamente a menos que los niveles de exposición sean muy altos y, por lo general, no están enlistados como enfermedades reportables. ${ }^{2}$ Por consiguiente, con la finalidad de evaluar y prevenir el envenenamiento infantil por plomo, es necesario realizar estudios epidemiológicos, cuyo diseño y población blanco van a diferir de acuerdo con la pregunta que se quiera contestar, el tiempo límite y los recursos disponibles.

\section{Evaluación del problema de salud pública}

El cuadro I presenta los pasos a seguir en la planeación de un estudio epidemiológico para la evaluación del envenenamiento por plomo. Después de establecer los objetivos es necesario definir tres elementos clave: a) las fuentes potenciales de exposición, b) los indicadores que se utilizarán para evaluar los efectos en la salud y la exposición del medio ambiente; y c) las estrategias de muestreo de la población en riesgo.

\section{Fuentes potenciales de exposición al plomo}

La exposición al plomo ocurre mediante diferentes fuentes y vías, sin embargo, las bases de datos sobre las fuentes potenciales de exposición, con frecuencia no se encuentran disponibles. Cuando las fuentes no están bien identificadas, los datos sobre el uso industrial del metal pueden proporcionar información sobre las fuentes potenciales de exposición. Por ejemplo, los datos sobre la operación de las fundiciones de plomo se pueden utilizar para determinar los sitios donde pudiese ocurrir una alta al plomo. Este metal se utiliza en muchos países para varios propósitos industriales como las pinturas, la soldadura, el barniz, la manufactura de baterías y como un aditivo para la

\section{Cuadro I \\ Pasos en la planeación de un estudio EPIDEMIOLÓGICO PARA LA EVALUACIÓN Del enVenenAmiento POR Plomo}

- Establecer los objetivos

- Determinar si existe información preliminar sobre las fuentes potenciales de exposición con la finalidad de enfocar el estudio

- Determinar los indicadores que se utilizarán para la evaluación, tanto de los efectos del plomo en la salud como de la exposición en el medio ambiente

- Determinar los factores que podrían afectar la medición del plomo en la sangre [grupo de edad, temporada del año, técnicas de medición (sangre venosa contra sangre capilar)]

- Definir la población que se va a estudiar, así como la estrategia de muestreo 
gasolina. ${ }^{3}$ Por lo cual, las vías de exposición que resultan de su uso pueden incluir el aire, la comida, el agua, el polvo, el suelo y la pintura. En situaciones específicas existen otras vías que pueden ser importantes como la cerámica tratada con barniz que contiene plomo, en México; o el «kohl» (polvillos negros empleados en la India para dar sombra a los ojos) y la medicina herbolaria $^{4}$ (figura 1).

Es importante mencionar que las fuentes y las vías de exposición al plomo pueden variar de una área a otra y de un país a otro. Por ejemplo, en los EUA la pintura con plomo es la fuente principal de exposición a este metal entre los niños. En México, esta pintura no se utilizó con frecuencia en las casas debido a que era más cara que la pintura de agua; sin embargo, la cerámica vidriada con plomo es la fuente principal de exposición. ${ }^{5}$

Marcadores biológicos de exposición al plomo y muestreo del medio ambiente

Para determinar el nivel de exposición al plomo se pueden utilizar diferentes marcadores biológicos que incluyen sangre venosa y capilar, sangre de cordón umbilical, plasma, orina, dientes, huesos y cabello. ${ }^{2} \mathrm{La}$ sangre, que refleja principalmente la exposición reciente, es el marcador biológico más comúnmente usado. El 99\% del plomo en la sangre se encuentra asociado con las células rojas y, como lo sugieren los estudios metabólicos, el plomo total en la sangre tiene una vida media de 25 a 30 días. El plomo en el plasma, que representa la fracción difusiva y biodisponible de éste que se encuentra en el cuerpo, posee el significado toxicológico más importante; ${ }^{6}$ no obstante, éste es difícil de medir con precisión y no se puede emplear como marcador biológico del envenenamiento por el metal en un sistema de vigilancia. La mayor parte de la carga de plomo en el cuerpo se almacena en los tejidos mineralizados. ${ }^{7}$ Sin embargo, aunque los niveles de plomo en los dientes y en los huesos reflejan una exposición acumulativa (durante varios años), no se pueden utilizar de manera rutinaria para la vigilancia.

De acuerdo con los patrones de los Centros de Control de las Enfermedades (Centers for Disease Control and Prevention $-\mathrm{CDC}$, por sus siglas en inglés-) los niveles de $\geq 10 \mu \mathrm{g} / \mathrm{dl}$ deben provocar algunas medidas preventivas a escala comunitaria. ${ }^{8}$ Sin embargo, los efectos de los niveles bajos, que incluyen deterioro en el desempeño cognoscitivo, cambios en el comportamiento y decremento en el cociente intelectual (inte-

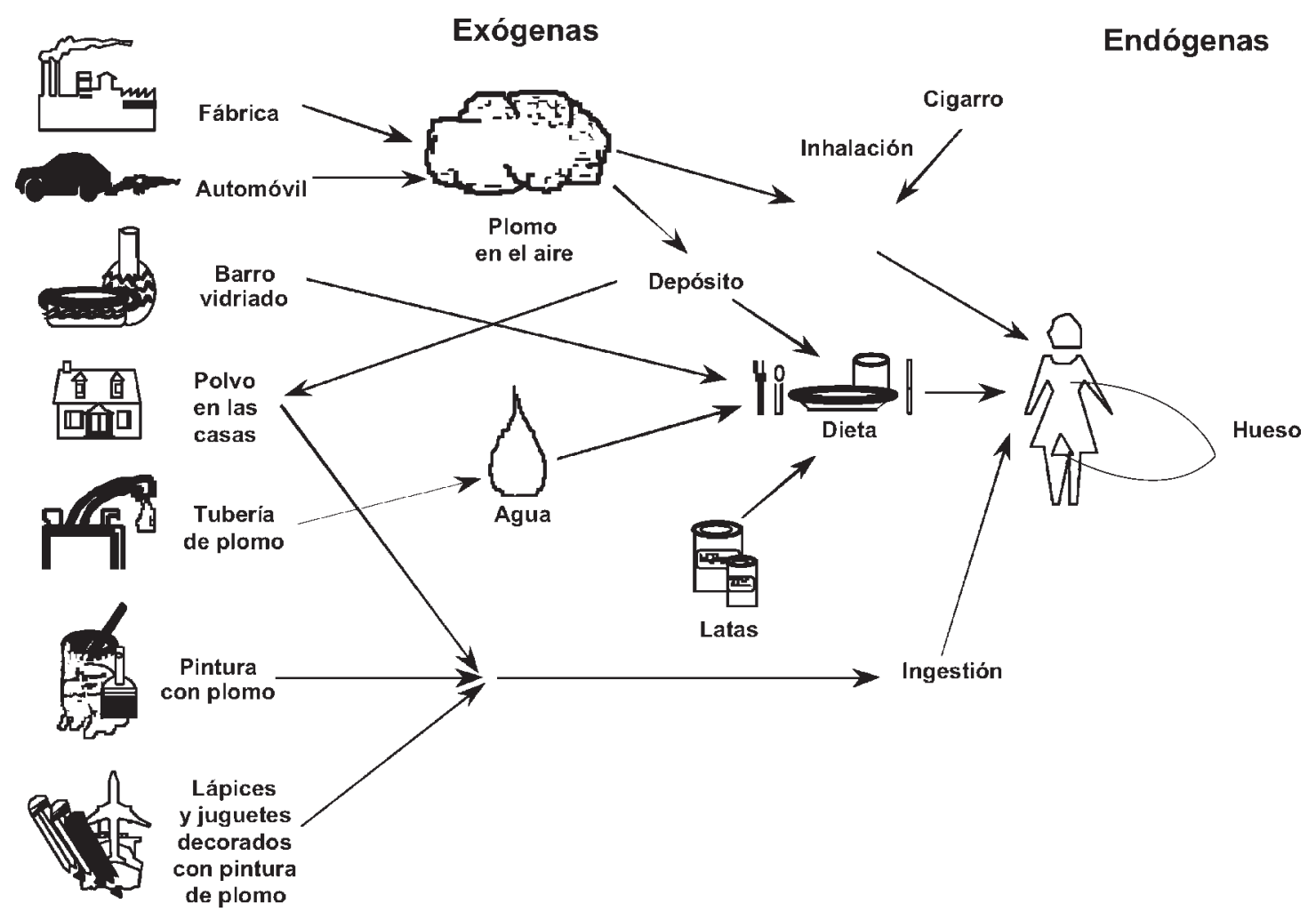

Figura 1. Fuentes de exposición a plomo 
lligence quotient-IQ, por sus sus siglas en inglés-) se han observado en niveles inferiores a $10 \mu \mathrm{g} / \mathrm{dl}$. Este descubrimiento está respaldado por muchos estudios hechos en diversas poblaciones. ${ }^{2,8}$

Si bien los cuestionarios pueden proporcionar alguna información acerca de las fuentes importantes de exposición al plomo, las muestras del medio ambiente son necesarias para confirmarlas. El conocimiento previo de las fuentes locales y las vías de exposición ayudará a enfocar el muestreo del medio ambiente. Para el plomo, dicho muestreo puede incluir el agua, el polvo, el suelo, la pintura, el aire, las cerámicas, los alimentos, y fuentes locales específicas de exposición como el "kohl" y la medicina herbolaria. Sin embargo, el análisis de estas muestras requiere de un laboratorio altamente calificado. Recientemente, el desarrollo de los analizadores portátiles permitió llevar a cabo los análisis del plomo en el medio ambiente y en la sangre en el lugar de estudio. Este equipo nuevo, a pesar de requerir una estandarización cuidadosa, permite a los trabajadores de campo una evaluación rápida de la contaminación por plomo.

\section{Estrategias de muestreo de la población en estudio}

Se pueden manejar varias estrategias de muestreo cuya selección depende de los objetivos del estudio, del tiempo límite y de los recursos disponibles. Cada una de ellas tiene sus ventajas y desventajas. El muestreo al azar con base en la población proporciona un estimado que se puede generalizar a una población más numerosa, pero requiere de un sistema de muestreo y la recolección puede llevar tiempo y ser costosa. El muestreo al azar, basado en las facilidades de los hospitales, las guarderías o las escuelas, es más sencillo y menos costoso para su ejecución; sin embargo, los resultados pueden ser menos representativos y estar propensos a sesgos (cuadro II). El muestreo objetivo de los grupos de alto riesgo, si es al azar, proporciona una estimación generalizable para el grupo. El muestreo de conveniencia (de voluntarios) es fácil, pero los resultados no se pueden generalizar con facilidad. Los reportes de los casos pueden conducir a la identificación, tanto de las poblaciones en riesgo como de las fuentes de exposición, y se les debe dar seguimiento mediante la investigación objetiva de la población identificada como de alto riesgo.

Factores que influyen en la medición del plomo en la sangre

$\mathrm{Al}$ interpretar los datos del plomo en la sangre es importante considerar los factores que pueden influir sobre la exactitud y la precisión de las mediciones. Los estudios en los cuales se compararon las medidas del plomo en las muestras de sangre capilar del dedo y en las muestras de sangre venosa, tomadas de los mismos individuos, muestran que, con una técnica adecuada de recolección, el promedio del sesgo positivo en una muestra de sangre capilar del dedo es menor de $1 \mu \mathrm{g} / \mathrm{dl}$. Esto es aceptable para los propósitos de investigación. Sin embargo, cuando no se cuenta con una técnica buena, las muestras de sangre capilar del dedo se contaminan y el sesgo positivo es mucho mayor. ${ }^{8}$ Los niveles individuales del elemento en la sangre también pueden variar con la edad y la temporada, por lo cual estos factores deben estar presentes al evaluar los cambios en los NPS durante el tiempo. También es importante considerar que el plomo se acumula en los huesos y que una exposición previa a este metal puede influir en su nivel actual en la sangre, debido a que

\section{Cuadro II \\ Estrategias de MUESTREO de LA POBLACIÓN EN ESTUdio}

Tipo de muestreo

Muestreo aleatorio basado en la población

Muestreo aleatorio basado en la facilidad (escuela, guardería, hospital)

Muestreo objetivo para los grupos de alto riesgo
Ventajas

Proporciona una estimación que puede ser generalizada para la población

Fácil de realizar, ya que la población es cautiva to por plomo es baja en la población general

Si se realiza de manera aleatoria, los resultados se pueden generalizar para el grupo

\section{Limitaciones}

Requiere de un sistema de planeación de muestreo Lleva tiempo y es costoso

Menos representativo, solamente se puede generalizar para la población estudiada Sujeto a prejuicio

Menos representativo, se puede generalizar sólo para el grupo estudiado

Si no se realiza de manera aleatoria, está sujeto a prejuicio

No se puede generalizar con facilidad

Sujeto a prejuicio 
éste se libera de los depósitos óseos. Como resultado, cuando una persona expuesta al plomo de manera crónica es trasladada del lugar de exposición, los niveles en su sangre tienden a disminuir lentamente con el tiempo. ${ }^{9}$

\section{Utilización de los datos de plomo en sangre para apoyar}

Los datos del plomo en la sangre se emplean para diferentes propósitos: la identificación de la población de alto riesgo, la identificación de las fuentes, la inspección de las tendencias, y el establecimiento de la relación entre la exposición y la respuesta. La siguiente sección presenta algunos ejemplos de cómo se pueden usar los datos sobre el plomo en la sangre para el esfuerzo preventivo.

\section{Identificación de la población de alto riesgo}

En un estudio hecho en una muestra aleatoria de niños escolares en Lima, Perú, para obtener los datos de referencia antes de la implantación de un programa de combustibles libre de plomo, los investigadores identificaron un sector en donde los niños tenían un NPS mayor al observado en otras partes de Lima. Una investigación más amplia en la misma población identificó un depósito abierto de minerales como la fuente principal de exposición al plomo en dicha población infanti1 ${ }^{10}$ (figura 2).

\section{Identificación de la fuente}

Para determinar las principales fuentes de exposición al plomo de madres y niños menores de cinco años de

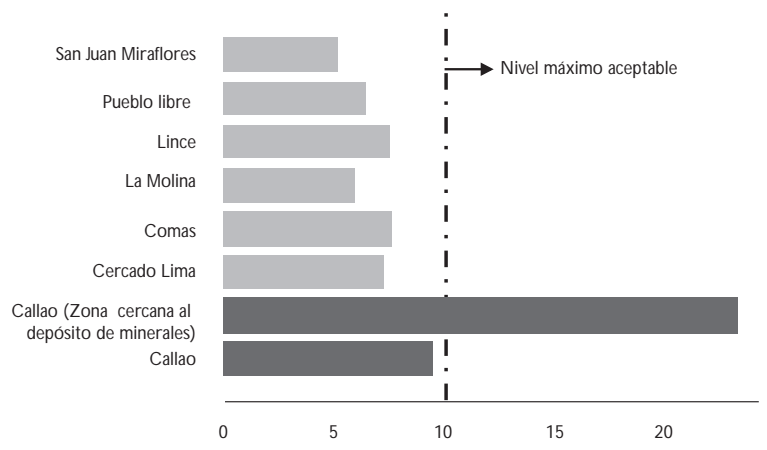

Referencia 10

Figura 2. Niveles de plomo en la sangre en diferentes zonas del área Metropolitana de Lima y El CALLAO, Perú, 1998-1999 edad se hizo un muestreo aleatorio, por medio del censo de las áreas y la selección al azar de las casas, basado en las poblaciones de dos áreas de la Ciudad de México: un área industrial y una residencial. ${ }^{11}$ Los NPS fueron ligeramente mayores entre los niños del área industrial y aumentaron con la edad. Las muestras del medio ambiente que con frecuencia excedieron las normas del plomo fueron las de la cerámica vidriada con plomo y las del polvo de la calle. Solamente $31 \%$ de las muestras de pintura excedió la norma. Los mecanismos principales, para la predicción de los NPS entre los niños, fueron el grado de exposición a la cerámica vidriada con plomo, la localización de la casa respecto del tráfico y la contaminación presente en las manos de los niños. Estos descubrimientos ayudaron a enfocar las acciones preventivas en las principales fuentes, entre las cuales se incluyeron la sustitución de los artículos con fondo de cerámica vidriada con plomo por vajillas metálicas, y la recomendación higiénica de que las manos de los niños se deben lavar varias veces al día.

Otro ejemplo de un muestreo aleatorio basado en la población es un estudio de la comunidad de fundidores en Avalos, Chihuahua. ${ }^{12}$ Después de la estratificación del área, de acuerdo con la distancia respecto de la fundición, se seleccionaron muestras aleatorias de mujeres en edad reproductiva $(n=45)$ y de niños de 1 a 5 años de edad $(n=45)$. Se obtuvieron tantas muestras de sangre como muestras del medio ambiente (suelo, agua, polvo y pintura de las casas). Las muestras de sangre y del suelo con mayor cantidad de plomo fueron las provenientes del área más cercana a la fundición. Como era de esperar, NPS más bajos se observaron entre los niños que vivían en las regiones más alejadas de la fundición, en donde el contenido de plomo en el suelo fue bajo (cuadro III).

El muestreo aleatorio basado en las facilidades también puede usarse para determinar las fuentes de exposición al plomo. La determinación de la exposición se realizó en niños de 6 a 9 años de edad que asistían a tres escuelas primarias localizadas a distancias diferentes $(650 \mathrm{~m}, 1750 \mathrm{~m}$ y $8100 \mathrm{~m}$ ) de los complejos de la fundición en México. ${ }^{13}$ Los valores más altos de plomo en la sangre, así como los niveles más altos de plomo en el suelo de las áreas de recreo, se observaron entre los niños que asistían a la escuela más cercana al complejo de fundición (cuadro II).

Estos dos estudios han proporcionado datos importantes para dar apoyo a la implantación de medidas de remedio y de control.

El reporte de los casos, o el agrupamiento de los casos de envenenamiento por plomo, también puede ayudar en la identificación de las fuentes de exposi- 
ción. Varios casos de envenenamiento ocurrieron entre niños de la embajada americana en México. ${ }^{14}$ Todos estos envenenamientos se produjeron en personas que habían estado en una fiesta en casa del embajador. Los investigadores encontraron una diferencia significativa de los NPS entre los niños que bebieron ponche en la fiesta y quienes no lo hicieron. El ponche se había servido en potes de cerámica vidriada con plomo. Los NPS más altos se observaron entre los niños que bebieron ponche en la fiesta y cuyos padres utilizaron cerámica vidriada con plomo para cocinar y servir los alimentos.

Además, en algunos casos de envenenamiento, se puede rastrear la ruta específica o la fuente de exposición con base en la relación del isótopo de plomo en la sangre del individuo, lo cual permite enfocar mejor la intervención ambiental (algunos ejemplos se presentan en este número especial). ${ }^{15}$

\section{Evaluación del cambio con el tiempo}

Para evaluar el cambio con el tiempo, los investigadores deben recolectar datos comparables en diferentes periodos. Existen tres formas: (a) con muestras aleatorias de la población (como se hizo en NHANES); (b) con muestras aleatorias de la facilidad (como en el sistema de vigilancia hecho por el Ministerio de Salud en México, basado en los NPS de cordón umbilical), y (c) con las muestras de conveniencia (como en los estudios entre seccionales repetidos entre los niños escolares, como se hizo en México).

Si comparamos los NPS en los tres estudios NHANES en los EUA, se observa una disminución general de 78\% en los NPS entre el estudio de 1976-1980 y el de 1988-1991. La disminución fue similar en todos los

\section{Cuadro III}

Concentración del plomo en las muestras

DE SANGRE Y DEL MEDIO AMBIENTE, DE ACUERDO Con diferentes estratos. Avalos, México, 1998

\begin{tabular}{ccrrrrr} 
Estrato* & $\begin{array}{c}\text { Pb en sangre } \\
\text { mujeres }\end{array}$ & $\begin{array}{c}\text { Pb en sangre } \\
\text { niños g/dl }\end{array}$ & $\begin{array}{c}\text { Suelo } \\
\text { ppm }\end{array}$ & $\begin{array}{c}\text { Polvo } \\
\mathrm{mg} / \mathrm{mtra}\end{array}$ & $\begin{array}{c}\text { Pintura } \\
\mathrm{ppm}\end{array}$ & $\begin{array}{c}\text { Agua } \\
\mathrm{ppb}\end{array}$ \\
I & 5.2 & 16.6 & 2113 & 87.7 & 1968 & $<2$ \\
\hline II & 6.3 & 8.9 & 225 & 71.0 & 1009 & $<2$ \\
\hline III & 4.3 & 7.7 & 205 & 30.0 & 390 & $<2$
\end{tabular}

Referencia 12

* Estrato I: 600 a 1200 m de la fundición Estrato II: 1201 a $1800 \mathrm{~m}$ de la fundición Estrato III: 1801 a 2400 m de la fundición grupos de edades. Las principales fuentes de exposición reducidas fueron el plomo en la gasolina (el cual disminuyó $99.8 \%$ de 1976 a 1990) y el porcentaje de latas de alimentos y bebidas suaves manufacturadas en los EUA que contenían soldadura de plomo (el cual disminuyó de $47 \%$ en 1980 a $0.9 \%$ en 1990). ${ }^{16-17}$

En México, el plomo presente en la sangre de cordón umbilical se investigó en muestras aleatorias provenientes de mujeres cuyos partos fueron atendidos en los centros públicos de salud. Los niveles de este elemento han disminuido con el tiempo, principalmente por la introducción de la gasolina sin plomo, lo que dio como resultado la disminución de las emisiones de este metal. Efectos similares se observaron entre los niños escolares ${ }^{18}$ (figuras 3 y 4 ).

\section{Establecimiento de la relación entre la exposición y la respuesta}

Una de las principales fuentes de plomo que existe aún en la población mexicana es el uso de la cerámica vidriada para preparar y almacenar alimentos. Varios estudios demostraron que los niños que ingieren alimentos preparados en este tipo de utensilios pueden presentar NPS de 3 a $4(\mu \mathrm{g} / \mathrm{dl})$, más altos que sus homólogos. Estos datos pueden sustentar el uso de cuestionarios específicos como alternativa para identificar a las poblaciones de alto riesgo, además de establecer medidas preventivas.

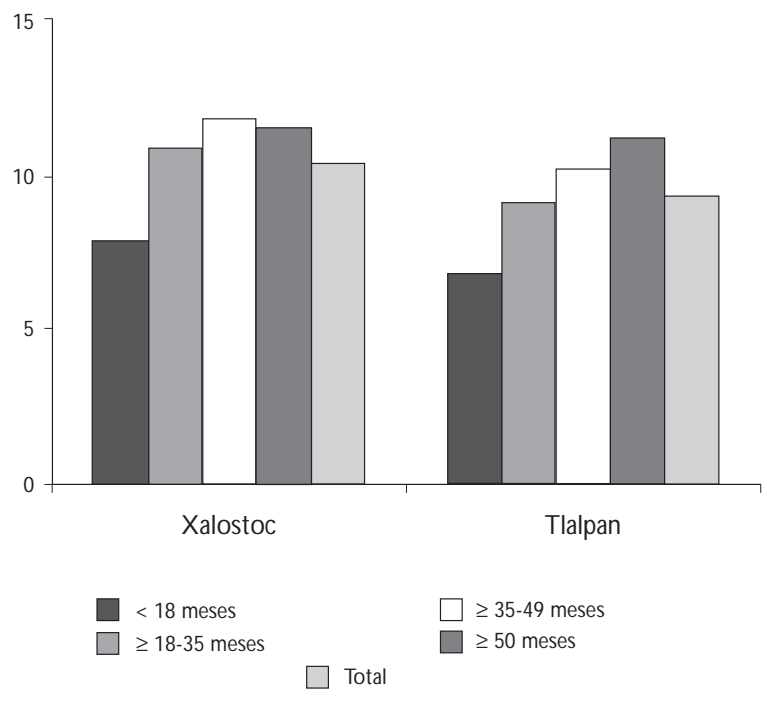

Figura 3. Niveles de plomo en la SANGRe ( $\mu \mathrm{G} / \mathrm{DL}$ ), DE acuerdo con la edad, en dos áreas de la CiUdad De México 


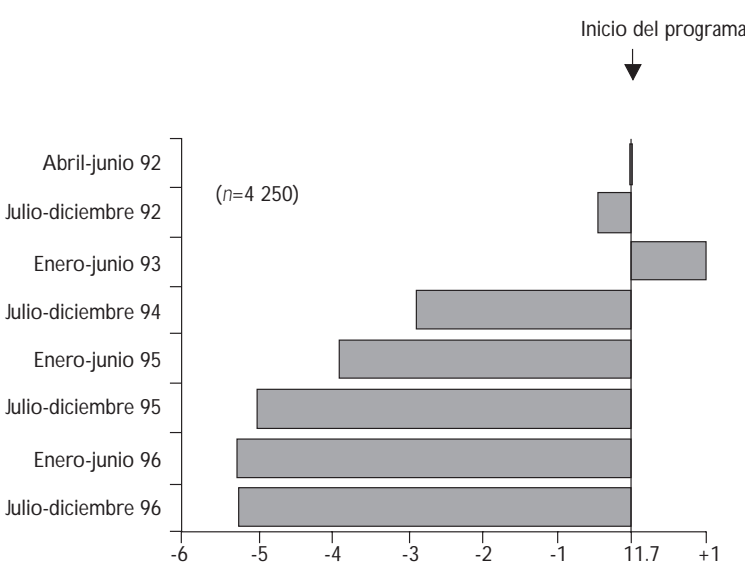

Figura 4. Niveles de plomo en los cordones umbiliCALES $(\mu \mathrm{G} / \mathrm{DL}$ ) PROVENIENTES DE MUJERES CUYOS PARTOS FUeron atendidos EN CLÍNICAS DE SALUd EN LA CiUdAd DE MÉxICO dE 1992 A 1997

Para algunas otras fuentes, como el plomo en el suelo, la relación entre la exposición y la respuesta puede ser más difícil de establecer pues la disponibilidad biológica del metal difiere dependiendo de la calidad del suelo. Sin embargo (The Environmental Protection Agency, EPA) ha desarrollado algunos modelos biocinéticos que permiten la predicción de los NPS de la población con base en los niveles observados en las muestras del medio ambiente. ${ }^{19}$ En los EUA los niveles de plomo en el polvo de la casa (procedente sobre todo de la pintura) se han relacionado con los NPS en un nivel tan bajo como $10 \mu \mathrm{g} / \mathrm{ft}^{2}$. Estos datos permitieron la utilización de la medición del plomo en el polvo como una alternativa para la investigación enfocada a la identificación de los ambientes peligrosos del hogar. ${ }^{9}$

Temas específicos

Para todos los diseños de muestreo, la información debe incluir la colección de las muestras biológicas y el uso de un cuestionario para recolectar la información general sobre los participantes y sobre las fuentes potenciales locales de exposición. Al concebir la estrategia de muestreo, los investigadores necesitan tomar varias decisiones:

- La edad del grupo por analizar es una decisión que dependerá de las fuentes potenciales de exposición. En los EUA los niveles de plomo más

altos se observan entre niños de dos años de edad, debido a que la fuente principal de exposición de éstos es por medio de las hojuelas de pintura. En otros países, como en México, donde otras fuentes son importantes, los NPS aumentan con la edad y se estabilizan

- Cuándo se debe realizar el muestreo, debido a que la temporada puede ser un factor que influye en la exposición al plomo

- El manejo del porcentaje de falta de respuesta, importante en la interpretación y generalización de los resultados

Además, con la finalidad de que la prevención del envenenamiento infantil por plomo sea efectivo, la determinación de los NPS debe involucrar a inversionistas y estar integrada dentro de una estrategia general que comprenda los diferentes pasos para el diagnóstico, el tratamiento, la identificación de las fuentes, la comunicación del riesgo, el control del medio ambiente y la implantación de medidas preventivas. De hecho, la prevención primaria debe ser el objetivo principal de los programas de prevención del envenenamiento infantil por plomo, y debe enfocarse a la identificación y al control de las fuentes de exposición en un nivel comunitario. El cuadro IV presenta las actividades a desarrollar dentro de la intervención en la comunidad para asegurar un programa exitoso. ${ }^{8}$

\section{Cuadro IV \\ Manejo de los peligros del plomo EN LA COMUNIDAD}

Actividades

Inspección y vigilancia

Determinación del riesgo y planeación integrada de prevención

Alcance y educación

Desarrollo de la infraestructura

Reducción del peligro
Definición

Determinar cuál es la población de riesgo y localizar la peor exposición

Analizar la información disponible para la determinación de las normas, para las fuentes de exposición al plomo y la población de alto riesgo, y desarrollar la prevención primaria

Informar a los encargados de los cuidados de la salud, a los padres, a los dueños de las propiedades y a otros inversionistas so bre la prevención del envenenamiento por plomo

Hacer un informe de los recursos necesarios para un programa exitoso de reducción del riesgo

Reducir los peligros de la exposición al plomo en el medio ambiente

Adaptado de la referencia 8 


\section{Conclusiones}

Se mostró que el plomo afecta a la salud y que el monitoreo biológico y del medio ambiente son medios importantes para la obtención de datos sobre la magnitud del problema en la población. Es importante definir claramente la población que se va a estudiar, utilizar la estrategia de muestreo adecuada así como considerar los factores que podrían afectar los NPS. Los estudios epidemiológicos deben incribirse en una estrategia general para la prevención y el control de la toxicidad del plomo.

\section{Referencias}

1. Stroup NE, Zack MM, W harton M. Sources of routinely collected data for surveillance. En:Teutsch SM, Churchill RE, Ed. Principles and practice of public health surveillance. $N$ ueva York (N Y), 0 xford: 0 xford University Press, 1994:31-95.

2.U S D epartment of Health and Human Services. Toxicological profile for lead.Atlanta (GA):A gency for Toxic Substance and D isease Registry, February 17, 1998.

3. Romieu I, Lacasaña M, McC onnell R, Research Group of the Pan A merican $\mathrm{H}$ ealth $\mathrm{O}$ rganization. Lead exposure in Latin A merica and The Caribbean. Environ Health Perspect 1997;105:398-405.

4. Romieu I, Palazuelos E, Hernández-A vila M, Ríos C, Muñoz I, Jiménez $C$ et al. Sources of lead exposure in Mexico City. Environ Health Perspect 1994;102:334-339.

5. Hernández-Avila M, Romieu I, Ríos C, Rivero A, Palazuelos E. Lead glazed ceramics as major determinants of blood lead levels in Mexican women. Environ Health Perspect 1991;94:117-120.

6. Smith D, Hernández-Avila M,Téllez-Rojo MM, Hu H. Relationship between lead in plasma and whole blood in women. Environ Health Perspect 2002;110:263-268.

7. Sanín LH, G onzález-C ossío T, Romieu I, Hernández-Avila M. Acumulación de plomo en hueso y efectos en la salud. Salud Publica Mex 1998;40(4):359-368.
8. US D epartment of Health and Human Services, Public Health Services, Centers for Disease Control and Prevention 1991. Preventing lead poisoning in young children. Atlanta (GA), 10/01/91.

9. Matte T. Interpretation and use of blood lead data. En: lead poisoning, prevention and treatment: Implementing a national program in developing countries. Proceedings of the international conference on lead poisoning prevention and treatment. 1999 Feb 8-10; Bangalore, India: 95-97.

10. Espinoza R, N arciso J, C arbajal L, Hernández-A vila JE, C astanaga C, Moscoso $S$ et al. Determinants of blood lead levels in children in Lima Metropolitan area. Salud Publica Mex 2003;45 (Suppl):

11. Romieu I, C arreón T, López L et al. Environmental urban lead exposure and blood lead levels in children of Mexico City. Environ Health Perspect 1995;103:1036-1040.

12. $O$ rnelas M, Sanín LH, Romieu I, Díaz-Barriga F, Reza S. Evaluación del riesgo de la intoxicación por plomo en la zona aledaña al fundidor de Avalos, Chihuahua. Reunión Anual de la AFMES; 1999 junio 2-4; San Antonio, Texas, EUA.

13. Gracia-Vargas GG, Rubio-Andrade M, D el Razo LM, Borja-A burto V, Vera-A guilar E, Cebrián ME. Lead exposure in children living in a smelter community in Region Lagunera, Mexico. J Toxicol Environ Health A 2001;62:417-429.

14. Matte TD, Proops D, Palazuelos E, G raef J, Hernández-Avila M. A cute high-dose lead exposure from beverage contaminated by traditional Mexican pottery. Lancet 1994;344(8929):1064-1065.

15. Chaudhary-W ebb M, Paschal D, Elliot C, Hopkins H, G hazi AM, Ting $\mathrm{H}$ et al . D etermination of lead isotope ratios in whole blood, pottery, and leaded gasoline: Lead sources in Mexico City.Atomic Spectroscopy 1998;19:156-163.

16. Centers for Diseases Control and Prevention. Blood lead levelsUnited States 1988-1991. MMW R; Morb Mortal W kly Rep 1994;43(30):141-145.

17. Centers for D iseases Control and Prevention. U pdate: Blood lead levels-United Sates 1991-1994. M MW R Morb Mortal W kly Rep 1997;46(7):545-547.

18. Hernández-Avila M. El plomo: un problema de salud pública en México. Intoxicación por plomo en México: prevención y control. En: Hernández-Avila M, Palazuelos-Rendón E, ed. Cuernavaca, Morelos, México: Instituto N acional de Salud Pública 1995; Perspectiva en Salud Pública 21:13-24.

19. Technical support document: Parameter and equations used in the integrated exposure uptake biokinetic (IEUBK) model for lead in children. Publication N umber 9285.7.22, EPA 540/R-94/040, EPA W ashington, DC, 1994. 